\title{
RECURRENT MENINGIOMA AFTER INITIAL RESECTION SURGERY - CASE REPORT
}

\author{
Ioana Cociasu', Irene Davidescu ${ }^{1,2}$, Ioan Buraga ${ }^{1,2}$, Bogdan O. Popescu ${ }^{1,2,3}$ \\ ${ }^{1}$ Department of Neurology, Colentina Clinical Hospital, Bucharest \\ 2 "Carol Davila" University of Medicine and Pharmacy, Bucharest \\ ${ }^{3}$ Laboratory of Molecular Medicine and Neuroscience, \\ "Victor Babes" National Institute of Pathology, Bucharest
}

\begin{abstract}
The most common tumours of the central nervous system, meningiomas are frequently diagnosed by accident when patients undergo imaging studies of the brain for other reasons. Most patients lack symptoms and thus can live their whole lives without knowing they have a brain tumour. Less fortunate patients seek medical advice for troubling symptoms - like seizures or disturbances of the cranial nerves - get surgery for the excision of the tumour and years later find out their tumour has come back. We are presenting the case of such a patient with a recurrent parietal meningioma.
\end{abstract}

Keywords: meningioma, multiple meningiomas, recurrent meningioma, seizures, radiotherapy

\section{INTRODUCTION}

Meningiomas are the most frequent tumours of the central nervous system (CNS). They are often incidental findings, due to their slow growth and lack of, or minimal symptoms. They affect women more than men, and their incidence increases with age. Symptomatology is mainly due to mass effect on neighbouring structures. Diagnosis is established using neuroimaging studies such as CT and MRI, with a preference for the latter. Therapeutic options include surgery with complete tumour resection, radiotherapy or a combination of the two. Higher grade meningiomas are more prone to recurrence. $(1,2)$

\section{CASE REPORT}

We are reporting the case of a 71 year old woman admitted by the Emergency Department in the Neurology Clinic in February 2015, for diffuse headache, with onset 2 weeks prior to presentation and progressive worsening. The headache does not subside to classic pain medication.

The patient's history is significant for a right parietal meningioma diagnosed due to generalised seizures and fully excised in 1991. After surgery the patient continued to have generalised tonicclonic seizures. At the time of admission the patient was treated with under dosed levetiracetam (250 $\mathrm{mg}$ three times daily). The patient states the last seizure experienced was 3 weeks prior to presentation when she presented an episode of tonic-clonic seizures of unknown duration, with loss of consciousness and spontaneous urine emission. She has had 3 similar episodes in the last month.

Prior CT scan performed in April 2014 when the patient was admitted with similar symptoms shows a well defined, homogenous small lesion with measurements of $1.3 / 1.1 \mathrm{~cm}$ and with tissue density situated in the right parietal region with adherence to the dura.

Physical examination is unremarkable. Neurologic examination is positive for left facial palsy of central origin, slight left symmetric hemiparesis (4/5 MRC), generalised anxiety and mixed insomnia.

Blood samples are within normal parameters.

EEG is unremarkable with no pathologic waveforms. 


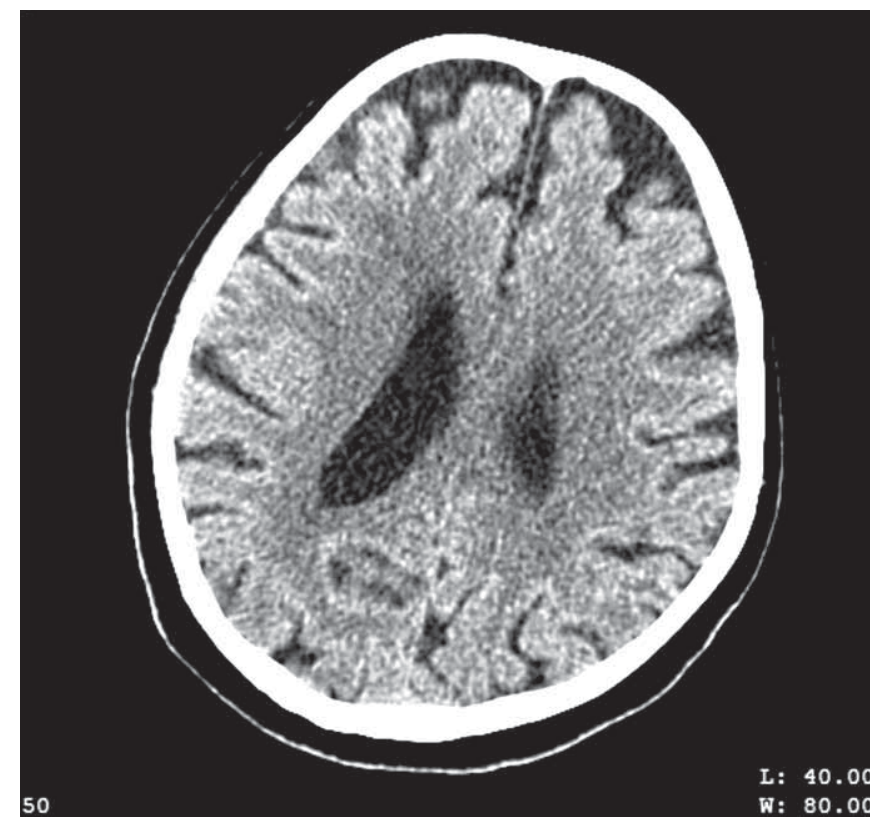

FIGURE 1. Native CT scan, axial sequence at the level of lateral ventricles, showing isodense globular shaped mass in the right parietal region, without surrounding oedema or mass effect on neighbouring structures. The scan also shows cortical atrophy and ventricular asymmetry due to faulty positioning.

Native CT scan shows signs of a right parietal craniotomy with bone flap. There is also a well defined mass; its density is similar to brain parenchyma, situated in the right parietal region, with a dural point of origin. Measurements of the tumour are $1.6 / 1.2 \mathrm{~cm}$. Contrast enhancement could not be performed due to patient's allergy to iodine based agents. The image is highly suggestive for a meningioma recurrence.

Taking in account the previous imaging studies, the tumour has grown in less than a year with approx. $0.3 \mathrm{~cm}$.

\section{DISCUSSION}

Meningiomas are the most common primary tumours of the CNS; they are usually extremely slow growing, benign and asymptomatic or minimally symptomatic. $(3,4)$ These tumours are often intracerebral but they can emerge anywhere from the dura, including the falx cerebri, tentorium cerebelli and venous sinuses. (2)

Women are more often affected than men, and the mean age of discovery is in the $7^{\text {th }}$ decade. (5) There have been several risk factors believed to increase the risk of developing a meningioma, among which radiation exposure (ionizing radiations and radiotherapy), genetic factors (type 2 neurofibro- matosis), hormonal factors (post-pubertal status in women, hormonal therapy and birth control), breast cancer and obesity. Historically, head trauma was first considered to be a risk factor in 1922 but current data does not support this hypothesis. Also use of mobile phones is also considered to be a risk factor but without any supporting evidence. (6-12)

Our patient has no positive history for any of the risk factors listed above, with the exception of her gender. She has no family history of meningiomas or any systemic malignancy.

The World Health Organisation (WHO) classifies meningiomas on morphologic criteria, from benign tumours - grade I, to atypical, clear cell and choroid meningiomas - grade II, to anaplastic, papillary and rhabdoid meningiomas - grade III. (13)

Many meningiomas are asymptomatic or minimally symptomatic. They are often incidental findings on neuroimaging studies or at autopsy. (4) Patients, when symptomatic, seek medical advice complaining of seizures or other focal deficits such as visual disturbances, auditory disturbances, mental status alteration or weakness. $(14,15)$

In the case of our patient, seizures were the initial complaint in 1991 when the first meningioma was diagnosed. After resection of the tumour, seizures diminished in frequency and intensity but did not subside completely. 
Neuroimaging studies, either CT or MRI with contrast enhancement, are sufficient for diagnosis and establishing treatment course; MRI is considered superior because of ability to show dural origin and relationship to surrounding structures. (16) Grading and a definite diagnosis require histological confirmation. On CT typical meningiomas have the appearance of well defined space occupying lesions, adjacent to dural structures and isodense with normal parenchyma. Calcifications are frequently found. When a contrast agent is administered, the mass is brightly enhanced. On MRI meningiomas appear isointense or hypointense on $\mathrm{T} 1$ and isointense or hyperintense on T2. (2) In the case of en plaque meningiomas, but not limited to, a characteristic finding is hyperostosis. (17)

When a patient presents more than one meningioma, either at the same time, or in sequence, either by recurrence or by postoperative seeding, this situation is referred to as multiple meningiomas. Epidemiology data show a prevalence of $1-15 \%$ in the general population, out of which an overwhelming proportion are women. (1)

When making a decision regarding treatment the physician needs to take in account several factors: whether the tumour is symptomatic or not, age and comorbidities of the patient, location and accessibility of the tumour, histological characteristics and the patient's opinion. One available option for asymptomatic patients is active surveillance, especially in older patients. The only curative treatment is complete surgical resection. (1) Younger patients should be screened for surgery due to high likelihood of tumour growth. First line therapeutic options include surgery, radiation therapy, or a combination of the two.

Recurrence rates are subject to a number of factors. One of these is the WHO grade, the higher the grade, the more likely it is the patient will have recurrence of the tumour. Another important factor is anatomic location and chance of complete surgical resection. Incomplete resections have high rates of recurrence. Another risk factor for recurrence is dural invasion or brain infiltration. A study conducted by Yamasaki et al found that the highest predictor for recurrence among grade I meningioma patients without documented multiple meningiomas or neurofibromatosis was tumour expression of vascular endothelial growth factor. (18)

Meningioma recurrences or incompletely resected tumours are treated with standard or stereotactic radiotherapy. Among these patients, risk of recurrence is high as well as systemic therapy complications such as radiation induced malignancy. Guthrie et al suggest radiotherapy should be used in the following instances: after the resection of a grade III meningioma, after an incomplete resection, in patients with multiple meningiomas in which surgery is no longer an alternative or in the case of primary inoperable tumours. (1)

There have also been multiple attempts for pharmacological treatment, most notable being: progesterone receptor inhibitors (mifepristone), chemotherapy agents (hydroxyurea, temozolomide), estrogen receptor inhibitors (tamoxifen), androgen receptor inhibitors, interferonalfa- $2 b$, somatostatin analogs (ocreotide) and molecularly targeted agents (platelet derived growth factor, epidermal growth factor, angiogenesis inhibitors). At present none have shown any significant efficacy. (19-28)

\section{CONCLUSIONS}

Meningiomas recurrence rates rise proportionally with grading. Meningiomas graded by the WHO classification as being II or above need to be screened for recurrence. Patients should be advised to seek medical advice whenever a change in the neurological status occurs or at a reasonable time period from initial surgery for neurological assessment. Early management of tumor recurrence might improve outcome in these patients.

\section{Acknowledgement}

This work is partially supported by the Sectoral Operational Programme Human Resources Development (HRD) financed by the European Social Fund and the Romanian Government through contract no. POSDRU 141531.

\section{REFERENCES}

1. Winn H.R. - Meningiomas. Youmans Neurological Surgery: Saunders; 2011: 1426-49.

2. Whittle I.R., Smith C., Navoo P., Collie D. - Meningiomas. Lancet 2004; 363(9420): 1535-43.

3. Annegers J.F., Schoenberg B.S., Okazaki H., Kurland L.T. - Epidemiologic study of primary intracranial neoplasms. Arch Neurol 1981; 38(4): 217-9.

4. Vernooij M.W., Ikram M.A., Tanghe H.L., et al. - Incidental findings on brain MRI in the general population. N Engl J Med 2007; 357(18): 1821-8.

5. Ostrom Q.T., Gittleman H., Liao P., et al. - CBTRUS statistical report: primary brain and central nervous system tumors diagnosed in the United States in 2007-2011. Neuro Oncol 2014; 16 Suppl 4: iv1-63. 
6. Banerjee J., Paakko E., Harila M., et al. - Radiation-induced meningiomas: a shadow in the success story of childhood leukemia. Neuro Oncol 2009; 11(5): 543-9.

7. Goutagny S., Kalamarides M. - Meningiomas and neurofibromatosis. J Neurooncol 2010; 99(3):341-7.

8. Preston-Martin S., Pogoda J.M., Schlehofer B., et al. - An international case-control study of adult glioma and meningioma: the role of head trauma. Int J Epidemiol 1998; 27(4):579-86.

9. Umansky F., Shoshan Y., Rosenthal G., Fraifeld S., Spektor S. Radiation-induced meningioma. Neurosurg Focus 2008; 24(5): E7.

10. Wiedmann M., Brunborg C., Lindemann K., et al. - Body mass index and the risk of meningioma, glioma and schwannoma in a large prospective cohort study (The HUNT Study). Br J Cancer 2013; 109(1):289-94.

11. Wiemels J., Wrensch M., Claus E.B. - Epidemiology and etiology of meningioma. J Neurooncol 2010; 99(3):307-14.

12. Cushing H. - The meningiomas (Dural endotheliomas): their source, and favoured seats of origin. Brain 1922; 45(2):282-316.

13. Louis D.N., Ohgaki H., Wiestler O.D., et al. - The 2007 WHO classification of tumours of the central nervous system. Acta Neuropathol 2007; 114(2):97-109.

14. Chozick B.S., Reinert S.E., Greenblatt S.H. - Incidence of seizures after surgery for supratentorial meningiomas: a modern analysis. J Neurosurg 1996; 84(3):382-6.

15. Anderson D., Khalil M. - Meningioma and the ophthalmologist. A review of 80 cases. Ophthalmology 1981; 88(10): 1004-9.

16. Mehdorn H.M. - Intracranial Meningiomas: A 30-Year Experience and Literature Review. Adv Tech Stand Neurosurg 2016; 43: 139-84.

17. Pieper D.R., AI-Mefty O., Hanada Y., Buechner D. - Hyperostosis associated with meningioma of the cranial base: secondary changes or tumor invasion. Neurosurgery 1999; 44(4): 742-6; discussion 6-7.

18. Yamasaki F., Yoshioka H., Hama S., Sugiyama K., Arita K., Kurisu K. - Recurrence of meningiomas. Cancer 2000; 89(5): 1102-10.
19. Ji Y., Rankin C., Grunberg S., et al. - Double-Blind Phase III Randomized Trial of the Antiprogestin Agent Mifepristone in the Treatment of Unresectable Meningioma: SWOG S9005. J Clin Oncol 2015.

20. Goodwin J.W., Crowley J., Eyre H.J., Stafford B., Jaeckle K.A., Townsend J.J. - A phase II evaluation of tamoxifen in unresectable or refractory meningiomas: a Southwest Oncology Group study. J Neurooncol 1993; 15(1): 75-7.

21. Wen P.Y., Quant E., Drappatz J., Beroukhim R., Norden A.D. Medical therapies for meningiomas. J Neurooncol 2010; 99(3): 365-78.

22. Chamberlain M.C., Johnston S.K. - Hydroxyurea for recurrent surgery and radiation refractory meningioma: a retrospective case series. J Neurooncol 2011; 104(3): 765-71.

23. Chamberlain M.C., Tsao-Wei D.D., Groshen S. - Temozolomide for treatment-resistant recurrent meningioma. Neurology 2004; 62(7): 1210-2.

24. Chamberlain M.C., Glantz M.J. - Interferon-alpha for recurrent World Health Organization grade 1 intracranial meningiomas. Cancer 2008; 113(8): 2146-51.

25. Simo M., Argyriou A.A., Macia M., et al. - Recurrent high-grade meningioma: a phase II trial with somatostatin analogue therapy. Cancer Chemother Pharmacol 2014; 73(5): 919-23.

26. Wen P.Y., Yung W.K., Lamborn K.R., et al. - Phase II study of imatinib mesylate for recurrent meningiomas (North American Brain Tumor Consortium study 01-08). Neuro Oncol 2009; 11(6): 853-60.

27. Norden A.D., Raizer J.J., Abrey L.E., et al. - Phase II trials of erlotinib or gefitinib in patients with recurrent meningioma. J Neurooncol 2010; 96(2): 211-7.

28. Lou E., Sumrall A.L., Turner S., et al. - Bevacizumab therapy for adults with recurrent/progressive meningioma: a retrospective series. J Neurooncol 2012; 109(1): 63-70. 